\title{
Stock Price and Index Forecasting by Arbitrage Pricing Theory-Based Gaussian TFA Learning
}

\author{
Kai Chun Chiu and Lei Xu \\ Department of Computer Science and Engineering \\ The Chinese University of Hong Kong, Shatin, N.T., Hong Kong, P.R. China \\ $\{$ kcchiu, lxu\}@cse.cuhk.edu.hk
}

\begin{abstract}
Viewed as a promising application of neural networks, financial time series forecasting was studied in the literature of neural nets and machine learning. The recently developed Temporal Factor Analysis (TFA) model mainly targeted at further study of the Arbitrage Pricing Theory (APT) is found to have potential application in the prediction of stock price and index. In this paper, we aim to illustrate the superiority of using the APT-based Gaussian TFA model as compared to three conventional approaches which are not financial model-based.
\end{abstract}

\section{Introduction}

The application of backpropagation networks in the prediction of stock prices was initiated by White [1] in 1988. Subsequent fruitful application of feedforword neural networks in stock price prediction was shown in [2-5]. The better performance of neural networks as compared to conventional statistical approaches in financial forecasting can be attributed to neural networks' capability to learn, adapt and generalize. Nonetheless, a typical weakness of feedforward neural networks is the inability to model existing temporal relations in financial time series. To overcome this limitation, recurrent neural networks with feedback were adopted in $[4,6]$.

From the perspective of statistical learning, stock price prediction was implemented via a special case of the alternative models for mixture of experts, called Extended Normalized Radial Basis Function (ENRBF), via the well-known Expectation-Maximization (EM) algorithm [7]. Furthermore, the concept of mixture of experts was further integrated with economic time series modelling, leading to the inception of the so-called mixture of Autoregressive Moving Average (ARMA) Models [8].

Still, all the above efforts failed to consider some well-known finance models which not only has established their foundation in the literature of economics and finance, but also has undergone rigorous statistical test concerning their explanatory power on certain empirically observed phenomena. Therefore it would be desirable that learning algorithms designed for financial forecasting also take advantage of those models. In literature, forecasting of stock prices within the framework of the Arbitrage Pricing Theory (APT) was discussed in [3]. Although it was a good initiative to adopt finance model in training neural networks for prediction, a major drawback is that the factors had to be assumed heuristically to be some items on the balance sheets of companies in the universe of U.K. stocks. 
Recently, a new technique aiming at the classical financial APT model and termed Temporal Factor Analysis (TFA) was proposed in [9] . In this paper, we consider how the APT-based Gaussian TFA model can be used for stock price and index prediction. Comparisons with some similar, previously adopted techniques are shown.

The rest of the paper is organized in the following way. Sections 2 and 3 briefly review the APT and the Gaussian TFA model respectively. Section 4 illustrates, via experimental comparisons, how Gaussian TFA can be applied to stock index forecasting. Section 5 concludes the paper.

\section{Review on Arbitrage Pricing Theory}

APT begins with the assumption that the $n \times 1$ vector of asset returns, $\tilde{R}_{t}$, is generated by a linear stochastic process with $k$ factors [10]:

$$
\tilde{R}_{t}=\bar{R}+A f_{t}+e_{t}
$$

where $f_{t}$ is the $k \times 1$ vector of realizations of $k$ common factors, $A$ is the $n \times k$ matrix of factor weights or loadings, and $e_{t}$ is a $n \times 1$ vector of asset-specific risks. It is assumed that $f_{t}$ and $e_{t}$ have zero expected values so that $\bar{R}$ is the $n \times 1$ vector of mean returns.

\section{Overview of Temporal Factor Analysis}

Suppose the relationship between a state $y_{t} \in \mathbb{R}^{k}$ and an observation $x_{t} \in \mathbb{R}^{d}$ are described by the first-order state-space equations as follows:

$$
\begin{aligned}
& y_{t}=B y_{t-1}+\varepsilon_{t}, \\
& x_{t}=A y_{t}+e_{t}, \quad t=1,2, \ldots, N .
\end{aligned}
$$

where $\varepsilon_{t}$ and $e_{t}$ are mutually independent zero-mean white noises with $E\left(\varepsilon_{i} \varepsilon_{j}\right)=$ $\Sigma_{\varepsilon} \delta_{i j}, E\left(e_{i} e_{j}\right)=\Sigma_{e} \delta_{i j}, E\left(\varepsilon_{i} e_{j}\right)=0, \Sigma_{\varepsilon}$ and $\Sigma_{e}$ are diagonal matrices, and $\delta_{i j}$ is the Kronecker delta function. Specifically, it is assumed that $\varepsilon_{t}$ is Gaussian distributed. The above model is generally referred to as the Gaussian TFA model. In the context of APT analysis, (1) can be obtained from (3) by substituting $\left(\tilde{R}_{t}-\bar{R}\right)$ for $x_{t}$ and $f_{t}$ for $y_{t}$. The only difference between the APT model and the TFA model is the added (2) for modelling temporal relation of each factor. The added equation represents the factor series $y=\left\{y_{t}\right\}_{t=1}^{T}$ in a multi-channel auto-regressive process, driven by an i.i.d. noise series $\left\{\varepsilon_{t}\right\}_{t=1}^{T}$ that are independent of both $y_{t-1}$ and $e_{t}$. Details about the TFA model and adaptive algorithms for its implementation can be found in [11].

\section{Using Gaussian TFA for Stock Index Prediction}

In this section, we aim to compare the relative performance of four similar approaches in financial prediction of stock indices. Based on the input source, they fall into two categories. Category I consists of the N-ENRBF and S-ENRBF approaches for which 
only time series of the respective stock/index data is supplied. Category II consists of the ICA-ENRBF and APT-based TFA-ENRBF approaches for which not only time series of the stock/index itself, but also data of the corresponding constituent stocks are involved. Since the constituent stock returns are used to recover market factors, approaches belonging to category II may be generally referred to as market-based approaches whereas those belonging to category I time series approaches. The following is a brief description of each approach.

- N-ENRBF Approach The adaptive ENRBF algorithm in [7] is used. The input vector consists of nonstationary raw index prices and is set as $\mathbf{x}_{\mathbf{t}}=\left[p_{t-1}, p_{t-2}\right.$, $\left.p_{t-3}\right]^{T}$ at time $t$.

- S-ENRBF Approach Quite similar to the previous approach, the adaptive EN$\mathrm{RBF}$ algorithm is adopted. The input vector at time $t$ is $\mathbf{x}_{\mathbf{t}}=\left[\tilde{R}_{t-1}, \tilde{R}_{t-2}, \tilde{R}_{t-3}\right]^{T}$, where stationary index returns $\tilde{R}_{t}$ are used instead of nonstationary index prices $p_{t}$. The index price at time $t$ can be recovered by from the predicted returns via $p_{t}=p_{t-1}\left(1+\tilde{R}_{t}+\bar{R}\right)$. Please refer to section 4.2 for the definition of $\tilde{R}$ and $\bar{R}$.

- ICA-ENRBF Approach This approach consists of two steps. First, the inverse mapping $\mathbf{y}_{t}=W \mathbf{x}_{t}$ is effected via the technique called Independent Component Analysis (ICA) for higher-than-second order dependence reduction. For this step the stock returns of the corresponding index constituents at time $t-1$ are used as input to recover independent components $\mathbf{y}_{t-1}$. Then, the adaptive ENRBF algorithm is adopted for establishing the relationship between $\mathbf{y}_{t-1}, x(t-1)$ and $x(t)$. In implementation, the Learned Parametric Mixture based ICA (LPM-ICA) algorithm [12] is used in view of its ability to separate any combination of sub-Gaussian and super-Gaussian source signals.

- APT-Based TFA-ENRBF Approach This approach differs from the preceding approach only in the first step. Here the Gaussian TFA algorithm instead of the LPM-ICA algorithm is used to recover independent hidden factors $\mathbf{y}_{t-1}$ at time $t-1$ from cross sectional stock returns $\mathbf{x}_{\mathbf{t}-\mathbf{1}}$. According to our previous work [13], the number of factors determined via the model selection ability of TFA is found to be 4 for HSI constituents and 3 for HSCCI constituents.

Account of experiments related to the first three approaches can be found in $[7,11]$. Here we focus on using the fourth approach where the classical financial APT model is taken into account and prediction is effected via utilizing the hidden factors extracted from stationary time series of returns.

\subsection{Data Considerations}

The analysis are based on past Hong Kong stock and index data. Daily closing prices of three major stock indices as well as 86 actively trading stocks covering the period from January 1, 1998 to December 31, 1999 are used. The number of trading days throughout this period is 522. Of the 86 equities, 30 of them are Hang Seng Index (HSI) constituents, 32 are Hang Seng China-Affiliated Corporations Index (HSCCI) constituents, and the remaining 24 are Hang Seng China Enterprises Index (HSCEI) constituents. 


\subsection{Data Preprocessing}

Except for the first approach, both stock and index prices should be converted to stationary returns. The required transformation can be described in four steps as shown below.

Step 1 Transform the raw prices to returns by $R_{t}=\frac{p_{t}-p_{t-1}}{p_{t-1}}$.

Step 2 Calculate the mean return $\bar{R}$ by $\frac{1}{N} \sum_{t=1}^{N} R_{t}$.

Step 3 Subtract $\bar{R}$ from $R_{t}$ to get the zero-mean return.

Step 4 Let the result of above transformation be the adjusted return $\tilde{R}_{t}$.

\subsection{Experimental Results}

Experimental investigation is based on the performance of prediction of the three stock indices, the HSI, HSCCI and HSCEI, as well as one of the stocks, the HSBC Holding, which is also a HSI constituent. We use the first 400 data for training and the remaining 120 data for test. Both training and test are carried out in an adaptive fashion. The number of optimum hidden units is determined by the automatic model selection of Rival Penalized Competitive Learning (RPCL) algorithm [14]. Typical results of HSI, HSCCI, HSBC prices using the N-ENRBF, S-ENRBF, ICA-ENRBF and APT-based TFA-ENRBF approach are shown in Fig. 1(a)-(d), 2(a)-(d) and 3(a)-(d) respectively.

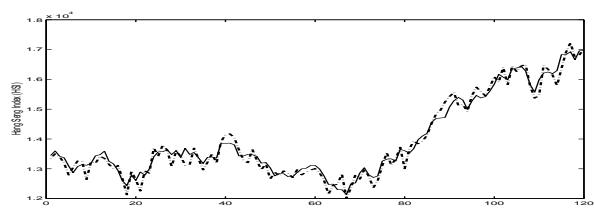

(a) By the N-ENRBF approach

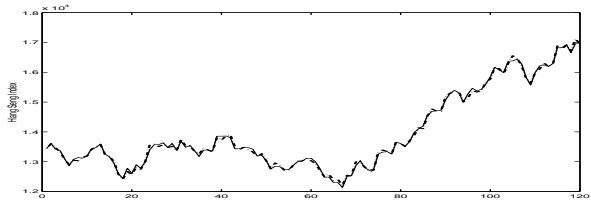

(c) By the ICA-ENRBF approach

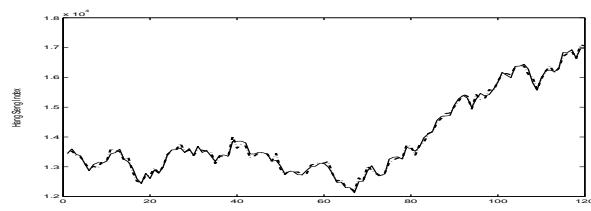

(b) By the S-ENRBF approach

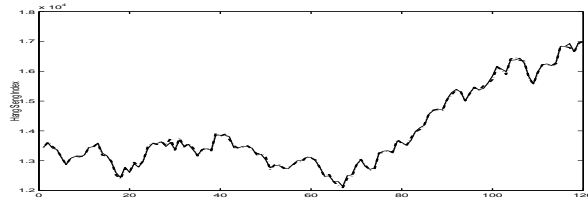

(d) By the APT-based TFA-ENRBF approach

Fig. 1. Result of prediction on HSI prices. “-” represents the desired signal while “-.” represents the predicted signal.

The performance of each method can be compared quantitatively by their respective Root Mean Square Errors (RMSE) between the predicted prices $\hat{p}_{t}$ and the desired prices $p_{t}$. As shown in Table 1, the APT-based TFA-ENRBF approach consistently outperforms the other three approaches by having the least RMSE for all three indices and the stock HSBC Holding. The ICA-ENRBF approach comes second and and the N-ENRBF approach the worst. 


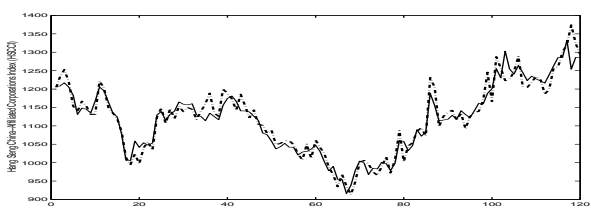

(a) By the N-ENRBF approach

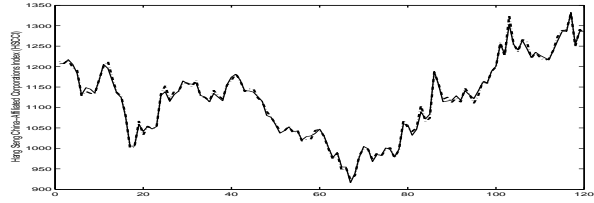

(c) By the ICA-ENRBF approach

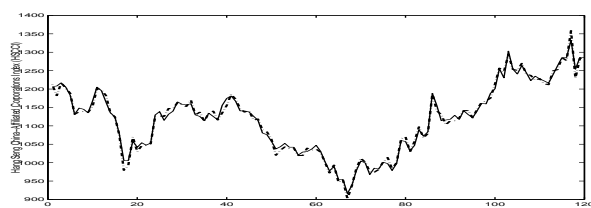

(b) By the S-ENRBF approach

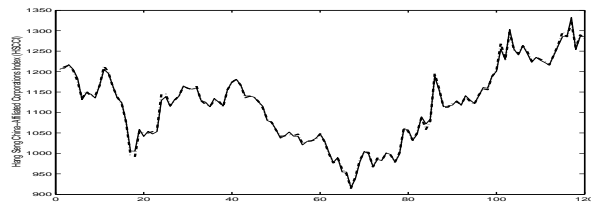

(d) By the APT-based TFA-ENRBF approach

Fig. 2. Results of prediction on HSCCI prices. “-” represents the desired signal while “-.” represents the predicted signal.

Table 1. Root Mean Square Error (RMSE) for different approaches

\begin{tabular}{|c|c|}
\hline Approach Type & HSI HSCCI HSCEI HSBC \\
\hline N-Adaptive ENRBF & $\begin{array}{lllll}232.9625 & 25.8021 & 9.9819 & 0.7957\end{array}$ \\
\hline $\mathrm{BF}$ & 648.72904. \\
\hline ICA-I & $\begin{array}{lllll}63.9681 & 6.0765 & 3.4340 & 0.3147\end{array}$ \\
\hline APT-based TFA-ENRBF & $\begin{array}{llll}47.6031 & 4.5202 & 2.2187 & 0.2346\end{array}$ \\
\hline
\end{tabular}

\subsection{Performance Evaluation}

Theoretically, the APT-based TFA-ENRBF approach is superior to the ICA-ENRBF approach because it takes into account the observation noise $e_{t}$ ignored by the LPMICA algorithm. The term $e_{t}$ in (3) of the TFA model speaks for itself. On the other hand, the ICA-ENRBF approach performs better than both the N-ENRBF approach and S-ENRBF approach because signals preprocessed by the ICA technique in general contain less redundancy. When viewed from an information perspective, both the SENRBF approach and N-ENRBF approach is lacking in information owing to the fact that no constituent stock returns are provided during parameter learning. Consequently, less precise parameters are estimated. It should be noted that in the N-ENRBF approach, nonstationary raw prices are used while in the other three approaches, stationary stock returns are used instead. Since nonstationary signals, such as the those referred to as random walks in finance, are in general more difficult to anticipate, this unfavorable condition makes the N-ENRBF approach the worst of all.

\section{Conclusion}

In this paper, we suggest how the Gaussian TFA model can be applied to stock price and index forecasting. We find that the APT-based TFA-ENRBF approach has demonstrated consistently superior performance over three other conventional approaches. 


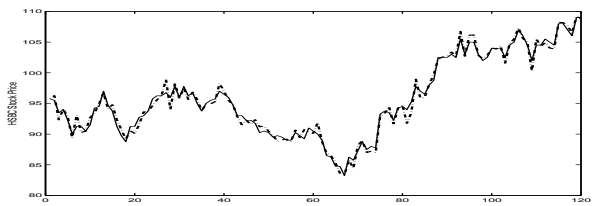

(a) By the N-ENRBF approach

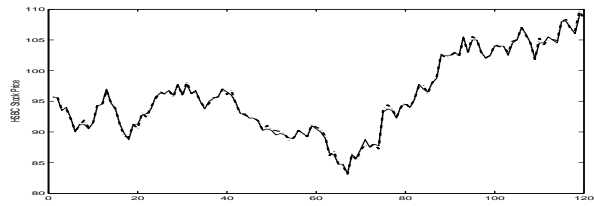

(c) By the ICA-ENRBF approach

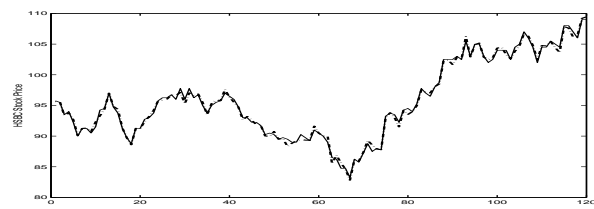

(b) By the S-ENRBF approach

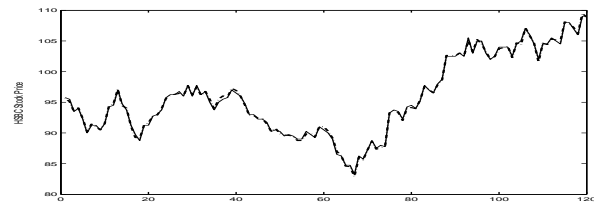

(d) By the APT-based TFA-ENRBF approach

Fig. 3. Results of prediction on the HSBC Holding stock prices. "-” represents the desired signal while "-." represents the predicted signal.

\section{References}

1. White, H.: Economic prediction using neural networks: The case of ibm daily stock returns. IEEE Int. Conf. on Neural Networks (1988)

2. Schoneburg, E.: Stock prediction using neural networks. Neurocomputing 2 (1990) 17-27

3. Refenes, A.N., Azema-Barac, M., Zapranis, A.D.: Stock ranking: Neural networks vs multiple linear regression. IEEE Int. Conf. on Neural Networks 3 (1993) 1419-1426

4. Giles, C.L., Lawrence, S., Tsoi, A.C.: Rule inference for financial prediction using recurrent neural networks. Proc. of IEEE/IAFE Conf. of Comput. Intell. for Fin. Eng. (1997) 253-259

5. Sagar, V.K., Lee, C.K.: A neural stock price predictor using qualitative and quantitative data. Proc. of 6th Int. Conf. on Neural Information Processing (ICONIP'99) 2 (1999) 831-835

6. Pantazopoulos, K.N., et al.: Financial prediction and trading stragegies using neurofuzzy approaches. IEEE Trans. on Systems, Man and Cybernetics 28 (1998) 520-531

7. Xu, L.: RBF nets, mixture experts, and Bayesian Ying-Yang learning. Neurocomputing 19 (1998) 223-257

8. Kwok, H.Y., Chen, C.M., Xu, L.: Comparison between mixture of ARMA and mixture of AR model with application to time series forecasting. Proc. of 5th Int. Conf. on Neural Information Processing (1998) 1049-1052

9. Xu, L.: Temporal BYY learning for state space approach, hidden markov model and blind source separation. IEEE Trans. on Signal Processing 48 (2000) 2132-2144

10. Ross, S.: The arbitrage theory of capital asset pricing. Journal of Economic Theory $\mathbf{1 3}$ (1976) 341-360

11. Xu, L.: BYY harmony learning, independent state space and generalized APT financial analyses. IEEE Transactions on Neural Networks 12 (2001) 822-849

12. Xu, L., Cheung, C.C., Amari, S.I.: Learning parametric mixture based ICA algorithm. Neurocomputing 22 (1997) 69-80

13. Chiu, K.C., Xu, L.: A comparative study of Gaussian TFA learning and statistical tests on the factor number in APT. to appear in Proc. of International Joint Conference on Neural Networks (IJCNN'02) (2002)

14. Xu, L., Krzyzak, A., Oja, E.: Rival penalized competitive learning for clustering analysis, RBF net, and curve detection. IEEE Trans. on Neural Networks 4 (1993) 636-649 